\title{
OSIRIS: a European project using a High Altitude Platform for forest fire monitoring
}

\author{
N. Lewyckyj, J. Biesemans \& J. Everaerts \\ Flemish Institute for Technological Research (VITO), \\ Remote Sensing \& Earth Observation Processes Unit (TAP), Belgium
}

\begin{abstract}
Within the framework of the European FP6 IST-5 project OSIRIS, a High Altitude Platform (HAP) will be used for the first time for surveying forest fires in the South of France. The HAP used will be the Mercator-1 Unmanned Aerial Vehicle (UAV) acquired by the Flemish Institute for Technological Research (VITO) in Belgium. Mercator-1, a solar aircraft able to remain for days at stratospheric altitudes (14 to $20 \mathrm{~km}$ ), will be used as a platform for a high resolution camera (GSD of a few meters). The platform will be controlled directly by an operator at a mobile Ground Control Station (GCS) or execute pre-defined flight planning (way points), which can be updated during flight. This will allow the end-users to optimize the image acquisition pattern as a function of the fire evolution. Because of its low airspeed, the platform is able to hover above a small region. Unprocessed data (level 0) will be down linked in real-time to the GCS for further processing (up to level 3 if required). Decision makers and/or the commanding officer of the fire brigades will thus have rapid access to up-to-date information at the intervention place. Furthermore, by means of a satellite uplink, the imagery will also be forwarded to a Central Data Processing Centre which is plugged into the OSIRIS Sensor Web. During the OSIRIS demonstration activities the benefits of the Sensor Web concept with respect to faster and/or better decision making will be evaluated.

The unmanned character of the platform does not put any pilots at risk and its long endurance allows the HAP to be used for the different phases of a disaster (from preparedness to recovery). Furthermore, the system will provide high resolution combined with frequent image update. A live demonstration is planned for spring 2009.
\end{abstract}

Keywords: disaster management, forest fire, remote sensing, real-time, high resolution, HAP, UAV, PEGASUS, OSIRIS. 


\section{Introduction}

The use of remote sensing techniques for disaster management is not new and it has increased in the last decade due to the increasing processing capacity of standard computers and to the availability of standard GIS packages. Also, the access to data is easier (via internet) and some data is even delivered for free. This is especially true for disasters (e.g. "the International Charter " Space and Major Disasters" [1]).

Conventional remote sensing data can be performed either by satellites or by airborne systems. Both technologies have their pro and contras.

Airborne remote sensing dates back to the $18^{\text {th }}$ century (using balloons). Airborne systems (helicopters and airplanes) offer great flexibility, short response times and are able to generate very high resolution data (typically few $\mathrm{cm}$ ). If digital sensors are used, they offer also the best performances in terms of spectral resolution and geo-referencing. All data that is acquired is available to the user as soon as the survey mission is completed.

They are however expensive to operate (costs for flying and maintaining an aircraft are considerable) and their operation is strongly limited by air traffic control constraints and by weather conditions. The coverage is also limited as typical swath (extend of the area observed on the ground level) are about few kilometres. Moreover, manned aircrafts cannot be used to fly in dangerous environment

Satellite remote sensing was first performed for civil purpose in the early 1970's. Satellites are very stable platforms that orbit the Earth in a predictable way. Polar orbits allow global coverage, with revisit times of a few days typically. They are therefore an ideal tool for the survey of large scale events (e.g. Tsunami in Asia 2004, large scale earthquakes or flooding events, big forest fires, ...). Beside the generally lower spatial resolution and positioning accuracy, the major disadvantages of current satellite systems is that (a) the overpass time is fixed since the trajectories can not be modified and (b) in cloud prone areas it can be quite difficult to obtain useable imagery (this does not apply for SAR sensors). All satellite systems deliver data in a digital format.

\section{Remote sensing using Unmanned Aerial Vehicles}

To compensate for the limitations of conventional systems, a third type of platform is gaining on importance: the Unmanned Aerial Vehicles (UAVs). UAVs, originally military systems developed for the so-called "dull, dirty or dangerous" tasks [2], are used for earth observation more and more. Applications include mapping, vegetation, risk management (fire, earthquakes, flooding,), environmental monitoring, local monitoring (archaeology, detection of oil spills, leakage in pipes, high voltage cable survey), etc. UAVs are also used for telecommunication. 
They can provide data with characteristics similar to those obtained using conventional manned aircrafts (high spatial resolution, good positioning accuracy and high spectral information). Because of their generally lower operational cost they even can provide higher temporal resolution (update rate) and they can be used also when weather conditions are not optimal (e.g. cloud coverage $>1 / 8$ ). Furthermore they can be deployed rapidly and are able to continuously survey the area of an event (still limited in time). These aspects are of major importance when considering disaster management. Finally, using UAVs avoids crews to be exposed to any harmful or dangerous situation (e.g. poor visibility due to smoke, dangerous environment, aircraft instability due to thermal gusts,).

However, UAVs have also drawbacks: in most cases, the UAVs used for civil applications are relatively small aircraft allowing only a limited payload mass. They are also limited in both autonomy (typically half an hour up to maximum few hours) and in available power supply for the sensors on board. Finally, a very important issue is the clearance (authorisation to fly) that is difficult to obtain, especially when flying in the same airspace as commercial aircrafts.

In recent years High Altitude Long Endurance (HALE) UAV or High Altitude Platform (HAP) have emerged. They represent about $4 \%$ of the total existing or under development UAV systems (EURO-UVS yearly report 2006, [3]) and have the potential of revolutionizing the remote sensing world. They fly above air traffic controlled airspace, in the stratosphere (between 12 and $20 \mathrm{~km}$ height) where the atmosphere has the lowest wind speeds and turbulences.

The first company to experiment with these concepts was Aerovironment (USA) breaking the world altitude record with the Helios Prototype that flew up to $96,863 \mathrm{ft}(29523 \mathrm{~m})$ altitude in August 2001[4]. Helios had a wingspan of 75 $\mathrm{m}$ (i.e. exceeding that of a Boeing 747) and was planned to stay at altitudes above $18 \mathrm{~km}$ for up to 6 months, powered by solar cells during day time, and regenerative fuel cells over night. It successfully demonstrated the use of this type of platform for telecom relay [5].

Sanswire Networks LLC has recently proposed to use a blimp, a so called "Stratellite", measuring $74.7 \mathrm{~m}$ in length for a total volume is $36800 \mathrm{~m}^{3}$. It is stated that it has a payload capacity of $1360 \mathrm{~kg}$, to provide wireless internet services over an area with more than $300 \mathrm{~km}$ radius [6]. It is planned to have 18 months endurance at $20 \mathrm{~km}$ altitude, using solar cells for power generation.

Other initiatives on these types of platforms include Sun-H2 [7] and a stratospheric airship under development by JAXA in Japan [8].

A number of studies in Europe, such as Heliplat at the Politecnico de Torino [9] and Solitair at DLR [10] have suggested different designs for this type of UAV. The latter one has rotating solar panels to optimally capture solar energy.

The advantages of a HALE UAV are clear: possible continuous operation in "mild" circumstances (so offering a stable platform) and less restrictions from air traffic control. This allows coverage of large areas as well as virtually continuous operation over a specific area. Also, the platform can be equipped with high resolution sensors, making it ideal complementary instrument to existing space borne and airborne systems. 


\section{The OSIRIS and the PEGASUS projects}

The OSIRIS project (Open architecture for Smart and Interoperable networks in Risk management based on In-situ Sensors) is a Sixth Framework integrated project of the European Commission in the area of Information Society Technology. It aims at (a) integrating a wide spectrum of sensor systems in a European Sensor Web and (b) testing and validating the quality of service of this Sensor Web in the framework of disaster management [11]. Existing stand-alone sensors (fixed and mobile) and sensor networks will be coupled in a self organizing and self healing way to provide the end-users a better tool-set for decision making. The project will demonstrate the performances of such system via four live demonstrations, one of them dealing with the survey of forest fires in South of France. Within this demonstration, different sensors will be used, including the PEGASUS system.

The PEGASUS project under development at VITO uses a HALE UAV able to operate at latitudes up to $52^{\circ}$ North between 14 and $20 \mathrm{~km}$ altitude for weeks to months without landing [12]. The system is powered by a combination of solar energy cells and batteries. In a later phase, batteries will be replaced by fuel cells. Despite its aptitude to cover up to $125.000 \mathrm{~km}^{2}$ on a yearly basis, the system can also continuously survey limited areas with a high update rate. This aspect is of major importance when dealing with disaster management.

In a first stage a very high resolution digital RGB camera (GSD of $30 \mathrm{~cm}$ ) called MEDUSA will be installed on the platform. The specification of the instrument has been defined on the basis of the requirements of commercial user community [13]. However, within the OSIRIS project the payload will be customized in order to best fit the requirements of the end-users, especially for forest fires.

The data will be directly down-linked to a mobile ground station and forwarded to a Central Data Processing and Archiving Center (CDPC) using a satellite uplink. After standard or on-demand processing, the collected data will be available for the stakeholders in near real-time via internet. The system will deliver data and information that are directly usable for programs like the Global Monitoring for Environment and Security (GMES) as well as for medium and small scale mapping applications (map scales typically 1:2500 - 1:20000). Within the framework of the OSIRIS project, the CDPC will be directly connected to the web-infrastructure to transfer the information to the end-users.

\section{Basic system requirements for forest fire survey}

According to the requirements extracted form the literature and from the enduser requirements as identified within the OSIRIS project, following needs were identified:

\section{Type of information:}

Fire detection (especially in the very early phase), view of starting fires in order to better react, dynamic evolution of the front fire, general view of the intervention teams for coordination purpose, survey of sub-urban regions for 
protection of the public, rescue team localisation and identification, view of the status of the infrastructure (roads,...), identification of burned areas, maps of temperature at the ground level for eventual restart of fires.

\section{Image resolution:}

Depending of the application described here above, between $0.5 \mathrm{~m}$ and $5 \mathrm{~m}$

Level of processing:

Raw image in the very early stage, geo-referenced images very rapidly.

Also composite images for general overview frequently.

\section{Temporal resolution:}

Composite images every 30 minutes, and local view in near real-time (few minutes delay) continuously

Spatial accuracy:

Spatial accuracy should be on the same order than image resolution

Delay for delivery:

Raw image in real-time (no delay), geo-referenced images within few minutes, general composite images every 30 minutes.

According to these requirements, following functions for the remote sensing sensor system can be derived for forest fire survey.

Platform: the remote sensing platform or platform constellation for the local monitoring and mapping is composed of High Altitude Platform(s) and/or Low Altitude UAV(s) and/or classical manned aerial survey platforms (helicopter, airplane). The remote sensing platform or platform constellation has flexible trajectories and shall have hovering capabilities to continuously update the area of interest, e.g. the moving fire line. Furthermore, the remote sensing platform or platform constellation shall have the ability to provide imagery continuously ( 24 hours/day) over the local region of interest (maximum region extent is estimated on some tens of square kilometres).

Payload: Depending on the information required, the remote sensing system shall sense the environment in thermal infrared or in visual bands. Thermal infrared allows for both day and night vision and provide relevant information despite smoke while visual bands are useful for "fuels mapping", for the survey of sub-urban regions, for the rescue of teams in difficulty, for the identification of remaining infrastructure.... The imagery is directly down-linked to the mobile ground station. This allows for real-time visual inspection of the incoming image information.

Ground Control Station (GCS): UAV platforms and their sensor systems are operated from a mobile ground control station. The possibility exists for the decision maker to inspect the incoming imagery in real-time in the mobile ground control station. The mobile ground control station also has a satellite uplink capability to forward the raw imagery to a high capacity central data processing centre (CDPC).

Central Data Processing Centre (CDPC): The CDPC contains the image processing computer infrastructure (a dedicated high performance computing 
cluster) for the archiving of the imagery and the production and archiving of the image products. The CDPC is the main interface with the end-user. Via a web interface, the users can query the database for new or old image products. Furthermore, via web services the CDPC can be plugged into a larger sensor system of systems.

The main product during a crisis is the area-wide composite of the fire line. This image product shall have a resolution of at least 5 meters, shall be delivered to the end-user in near real-time (update at least every 30 minutes), shall be delivered in a standard GIS format according user specifications and shall be delivered in an ortho-rectified format with a projection system and projection datum according user specifications. The image processing software system is installed at the CDPC.

\section{Logical model description}

Figure 1 present the layout of the logical model of the remote sensing system in support of local monitoring and mapping of forest fires within the framework of the OSIRIS project.

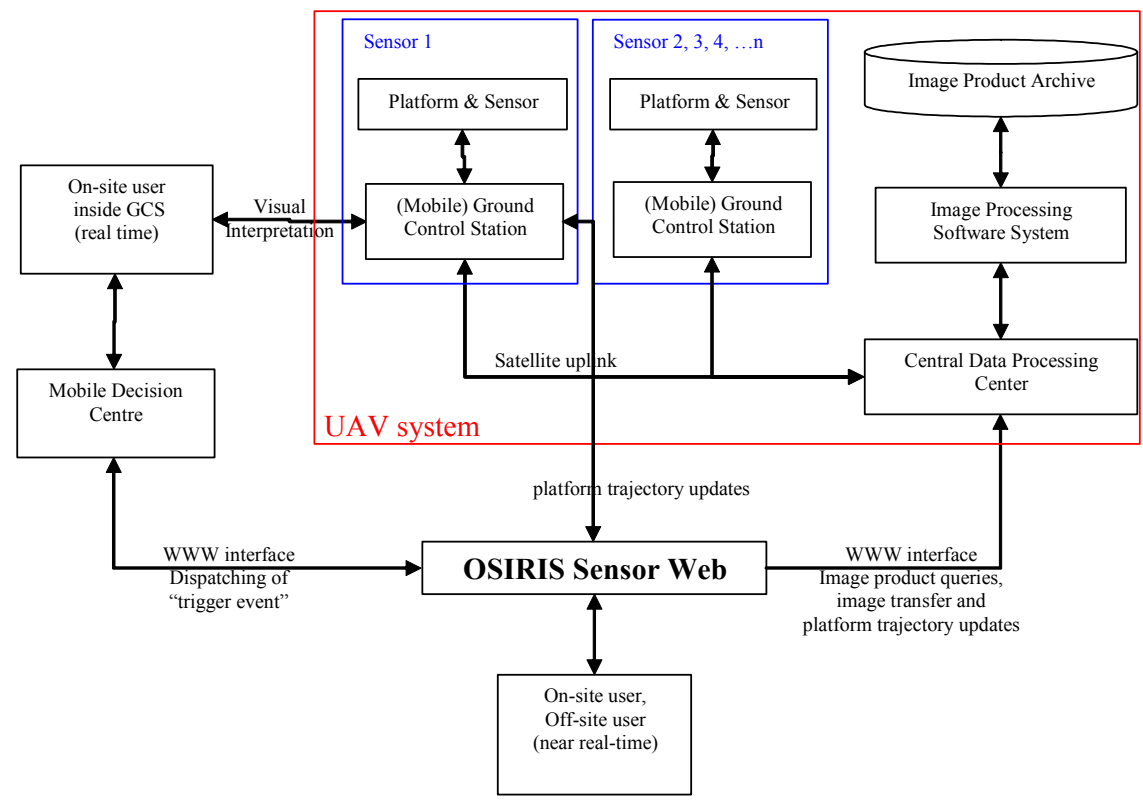

Figure 1: Logical model description of the remote sensing system in support of local monitoring and mapping of forest fires.

In this figure, the remote sensing platform or platform constellation is composed of High Altitude Platform(s) and/or Low Altitude UAV(s) and/or classical manned aerial survey platforms (helicopter, airplane). By means of a 
satellite uplink, the raw image data stream if pushed to the Central Data Processing Centre (CDPC), which contains the HPC (High Performance Computing) infrastructure to (a) process the raw imagery according the user specifications and to (b) archive the image products.

In time critical applications, it is possible for an on-site user to visually inspect the incoming imagery in the (mobile) ground control station. As such, the user can exploit the incoming information in his decision making process in realtime.

At the CDPC, the imagery is further processed into user products. This processing encompasses: (a) the archiving of the raw incoming image data stream, (b) the georeferencing and co-registration of the imagery, (c) the production of image composites of the fire line (the time coverage can be fully specified by the user and can be changed during the mission, e.g. 5 minute, 10 minute or 30 minute composites), and (d) the archiving of the user specific image products. All image products are referenced in the CDPC database system.

The on-site and/or off-site user has the possibilities to retrieve the image information via the OSIRIS Sensor Web.

When considering unmanned platforms it will also be possible for the users to specify the trajectory via waypoints. The UAV-pilot, positioned in the mobile ground control station, can validate this new trajectory and reprogram the platform. The request for a modification of the trajectory will be communicated via the OSIRIS web-architecture.

Interfaces between the $U A V$ system and the OSIRIS web architecture are thus present at three levels:

- at the CDPC to allow the end-users to request processing of data and to access archived data,

- at the mobile GCS to allow the end-users request an update of the platform trajectory, and

- via the mobile decision centre that will have a permanent link with an enduser present in the GCS to visualize in real-time the raw data.

\section{Validation exercise}

In order to demonstrate the added value of such information, a live exercise will be performed in real-time during the spring 2009 in the South of France. The scenario of the exercise will be defined in close collaboration with the end-users. During the so-called "ecobuage" period (period when small part of the forests are burned under the control of the French Firemen in order to better control potential forest fire during the summer) the PEGASUS system will be used. The HALE UAV will be equipped with the high resolution MEDUSA camera and will provide an image composite of the fire front, local high resolution images of sub-urban regions in the vicinity of the fire, high resolution imagery of the team (fireman trucks,...) involved in the intervention, a global view of the infrastructure in the vicinity of the fire... 
The issue of the fire detection will not be a task for the RS system as forest fire detection in France is already very efficient and it takes only few minutes with the existing infrastructure.

A small UAV equipped with a thermal camera will also possibly be used as a complementary source of information.

The data will be sent from the UAV systems to an autonomous mobile ground control station located in the vicinity of the mobile decision centre of the Fire brigades. A command officer will be present in the GCS for analyse the raw imagery in real-time de visu. After buffering of the data, some limited image processing will be performed at the level of the GCS. I $n$ the mean time, the raw data will be sent to the Central Data Processing Centre located in Mol, Belgium via satellite uplink for further processing and archiving. Via de OSIRIS webinfra-structure, the end-users will be able to request data and specific processing on-demand. Once processed, the information will be sent to the end-user who will have the possibility to ask for further processing and/or acquisition. The end-user will even have the opportunity to adapt the flying pattern of the UAV in real-time according to the situation evolution.

After this exercise, a debriefing will be performed together with the end-users to analyse the results and to propose possible improvements.

\section{Other applications}

Due to the intrinsic characteristics of the PEGASUS system (very high flexibility, rapid deployment, very high resolution data, real time character) the system is also suited for several other applications dealing with disaster management. High resolution data (up to $30 \mathrm{~cm}$ spatial resolution) to assess the extent of the damage in case of earthquake or tornados, large scale gas explosion or industrial accidents are some examples. Also the survey of active volcanos during the phase just before the explosion (mostly performed with manned system) or the determination of the exact extend of flooding (small to medium scale) are possible. HAPs can also be used for the preparation and survey of large scale events like e.g. Olympic Games or large Rock Concerts. More generally, HAP systems are suited for the survey of local to regional areas with a much higher update rate than any orbital satellites can offer: using a spatial resolution of $1 \mathrm{~m}$ the HAP can fully cover an circle area of approximately $70 \mathrm{~km}^{2}$ every minute, $\sim 300 \mathrm{~km}^{2}$ every 25 minutes or a square area of $60 \times 60 \mathrm{~km}^{2}$ daily. The major advantage is certainly the possibility to operate during hours, days or even weeks continuous survey above a limited area.

\section{Acknowledgements}

OSIRIS is an Integrated Project co-funded by the INSFO DG of the European Commission within the RTD activities of the Thematic Priority Information Society Technologies.

The PEGASUS initiative is supported by the Flemish Government (Belgium) that decided to fund the acquisition of the platform in 2005. 
The MEDUSA payload is designed and manufactured in the framework of the ESA contract number C90243.

\section{References}

[1] Charter (2007). The International Charter "Space and Major Disasters", http://www.disasterscharter.org/, accessed on 07 March 2007

[2] Aldridge, E.C., Stenbit, J.P. (2002). Unmanned Aerial Vehicles Roadmap 2002-2027, Office of the Secretary of Defense, Washington DC, USA.

[3] EURO-UVS (2006). http://www.uvs-international.org/, accessed on 07 March 2007.

[4] AeroVironment (2005). http://www.aerovironment.com/areaaircraft/unmanned.html, accessed on 07 March 2007

[5] Skytower (2005). http://www.skytowerglobal.com/begin.html, accessed on 07 March 2007

[6] Sanswire (2005). http://www.sanswire.com/stratellites.htm, accessed on 07 March 2007

[7] Sun-H2 (2005). http://www.sun-h2.be/en/index.htm, accessed on 07 March 2007

[8] Sasa, S. (2004). Stratospheric Platform Program in Japan and Ground-toStratosphere Flight Test. $9^{\text {th }}$ UAVNet Meeting, Amsterdam, 26-27 January 2004. http://www.uavnet.com/DL/Document_Library/ Amsterdam_Meeting/Stratospheric_platform_Sasa.pdf

[9] Tozer, T.C., Grace, D. (2001). HeliNet - The European Solar-Powered HAP Project, UVS Tech 2001, Euro UVS, Brussels, Belgium, December 2001.

[10] Friehmelt, H. (2003): SOLITAIR - A solar-powered HALE demonstrator. In: ONERA [Hrsg.]: Proceedings ODAS 2003, S. S2-2 -, ODAS 2003, Toulouse, France, June 4-6, 2003, ISBN 2-7257-0011-6.

[11] OSIRIS (2007). http://www.osiris-fp6.eu, accessed on 07 March 2007

[12] PEGASUS (2007). http://www.pegasus4europe.com, accessed on 07 March 2007

[13] Fransaer D, Vanderhaeghen F, Everaerts J (2004). PEGASUS: Business Plan for a Stratospheric Long Endurance UAV System for Remote Sensing, Proc ISPRS congress, Istanbul, Turkey, July 2004. 